\title{
The shear-Hall instability in newborn neutron stars
}

\author{
T. Kondić ${ }^{\text {, G. Rüdiger }}{ }^{1}$, and R. Hollerbach ${ }^{2}$ \\ 1 Astrophysikalisches Institut Potsdam, An der Sternwarte 16, 14482 Potsdam, Germany \\ e-mail: [gruediger; tkondic] aaip.de \\ 2 University of Leeds, Department of Applied Mathematics, Leeds LS2 9JT, UK \\ e-mail: rh@maths. leeds.ac.uk
}

Received 24 February 2011 / Accepted 3 October 2011

\begin{abstract}
Aims. In the first few minutes of a newborn neutron star's life the Hall effect and differential rotation may both be important. We demonstrate that these two ingredients are sufficient for generating a "shear-Hall instability" and for studying its excitation conditions, growth rates, and characteristic magnetic field patterns.

Methods. We numerically solve the induction equation in a spherical shell, with a kinematically prescribed differential rotation profile $\Omega(s)$, where $s$ is the cylindrical radius. The Hall term is linearized about an imposed uniform axial field. The linear stability of individual azimuthal modes, both axisymmetric and non-axisymmetric, is then investigated.

Results. For the shear-Hall instability to occur, the axial field must be parallel to the rotation axis if $\Omega(s)$ decreases outward, whereas if $\Omega(s)$ increases outward it must be anti-parallel. The instability draws its energy from the differential rotation, and occurs on the short rotational timescale rather than on the much longer Hall timescale. It operates most efficiently if the Hall time is comparable to the diffusion time. Depending on the precise field strengths $B_{0}$, either axisymmetric or non-axisymmetric modes may be the most unstable.

Conclusions. Even if the differential rotation in newborn neutron stars is quenched within minutes, the shear-Hall instability may nevertheless amplify any seed magnetic fields by many orders of magnitude.
\end{abstract}

Key words. instabilities - magnetohydrodynamics (MHD) - magnetic fields - stars: neutron

\section{Introduction}

The classical dynamo mechanism for generating cosmic magnetic fields is based on the inductive effects of rotating anisotropic turbulence. Because the same turbulence prevents uniform rotation from being a solution of the Reynolds equation, in the majority of dynamo models, the basic mechanism for producing toroidal fields from poloidal fields is differential rotation. However, it alone only produces toroidal fields that grow linearly in time, until the resulting Maxwell stresses suppress the differential rotation. To produce a self-excitation of magnetic fields as an instability that grows exponentially in time, another effect is needed to produce poloidal fields from toroidal ones.

There are many possibilities. The simplest one is a meridional flow. For certain combinations of differential rotation, meridional flows, and finite conductivity, the coupled system allows the self-excitation of (purely non-axisymmetric) fields (e.g. Dudley \& James 1989). It is also possible to close the circuit by the $\alpha$-effect, which represents the existence of an electromotive force parallel to the mean magnetic field. The result is a so-called $\alpha \Omega$-dynamo, which for a high magnetic Prandtl number can be modified by the action of meridional flow (Choudhuri et al. 1995; Küker et al. 2001). Driven turbulence subject to a shear flow in liquid metals is also able to work as a dynamo if the turbulence intensity is stratified in the direction orthogonal to the shear-flow plane (Rüdiger \& Kitchatinov 2005).

The dynamo equations resulting from purely hydrodynamic flows are all linear, and the amplification can start from arbitrarily weak seed fields. This is not the case, however, for possible dynamo mechanisms where the coupling $B_{\text {tor }} \rightarrow B_{\text {pol }}$ depends on magnetic instabilities. Such alternative mechanisms only work if the seed field is sufficiently strong. There are a variety of magnetic instabilities on which the dynamo mechanism can be based. Schmitt et al. (1996) started the construction of such models with their on-off dynamo model where the $\alpha$-effect only exists if the magnetic field exceeds a certain threshold value determined by the magnetic buoyancy instability. Other possibilities have been suggested on the basis of both the magnetorotational instability (MRI) and the Tayler instability (Spruit 2002).

In this Letter it is demonstrated that in the presence of a shear flow, including the Hall term in Ohm's law is enough to produce a "shear-Hall instability" (SHI). The Hall effect produces off-diagonal elements in the conductivity tensor that are of the first order in the magnetic field. The SHI is thus a magnetically nonlinear instability, but with the particularity that there is no threshold value. That is, if the shear is strong enough, the Hall effect, hence the initial seed field, can be arbitrarily weak.

The natural application of this shear-Hall instability is in newly born neutron stars. In the first few minutes of its life, the crust of the star can rotate differentially, as shown in a number of numerical computations of the supernova core (Mönchmeyer \& Müller 1989; Janka \& Moenchmeyer 1989; Dimmelmeier et al. 2002; Kotake et al. 2004; Ardeljan et al. 2005; Burrows et al. 2007). Differential rotation in the core of the collapsing progenitor will induce strong differential rotation in the newly formed neutron star (Ott et al. 2005).

Initially, the star cools down by neutrino emission. As a result of this cooling, the core becomes distinct from the outer layer, and the crust condenses at a temperature of a few $10^{9} \mathrm{~K}$ 
(Dall'Osso et al. 2009, and references therein). Any differential rotation can thus only exist in a limited time-interval that depends on the type of reactions causing the cooling. In the case of the direct URCA process, the cooling is extremely fast, with estimates giving a characteristic time of a few minutes (Page et al. 2006).

However, the very rapid rotation rates, with periods of order $10^{-2} \mathrm{~s}$, mean that even just a few minutes is long enough for the star to undergo several thousand revolutions. Furthermore, we show that the SHI grows on this very fast rotational timescale, so even though it only exists for a few minutes, this is sufficient for it to amplify a seed field by many orders of magnitude. Of course, the time evolution of the shear is excluded in this simplified model, so the exact amplitude of the field at the end of the SHI phase must still be deduced from full nonlinear simulations of the Hall-MHD system.

\section{The shear-Hall instability}

The Hall effect is important whenever a plasma is magnetized enough. The level of magnetization is determined by the Hall parameter, $R_{B}=\sigma B_{0} / n e c$, with $B_{0}$ a characteristic magnitude of the field, $\sigma$ the electric conductivity, $n$ the electron number density, $e$ the electron charge, and $c$ the speed of light. If the Hall parameter $R_{B}$ is large, the Hall effect is important.

In a neutron star, both the electron number density and the conductivity vary with depth. The conductivity also varies with temperature. To focus on the basic physics of the instability process, we neglect all such variations and treat both $n$ and $\sigma$ as constants. However variations in $n$ in particular can interact with the Hall effect in interesting and unexpected ways (Vainshtein et al. 2000; Hollerbach \& Rüdiger 2004).

The magnetic Reynolds number is $\mathrm{Rm}=R^{2} \Omega_{0} / \eta$, where $R$ is the star's radius, $\Omega_{0}$ the maximal angular velocity, and $\eta$ its magnetic diffusivity. Scaling length by $R$, time by $R^{2} / \eta, u$ by $R \Omega_{0}$, and $\boldsymbol{B}$ by $B_{0}$, the induction equation becomes

$$
\begin{aligned}
& \frac{\partial \boldsymbol{B}}{\partial t}= \\
& \quad-R_{B} \nabla \times((\nabla \times \boldsymbol{B}) \times \boldsymbol{B})-\nabla \times(\nabla \times \boldsymbol{B})+\mathrm{Rm} \nabla \times(\boldsymbol{u} \times \boldsymbol{B}) .
\end{aligned}
$$

We linearize this equation about the uniform axial field $\boldsymbol{B}_{0}=$ $\boldsymbol{e}_{z}$, yielding

$$
\begin{aligned}
& \frac{\partial \boldsymbol{B}}{\partial t}= \\
& \quad-R_{B} \nabla \times\left((\nabla \times \boldsymbol{B}) \times \boldsymbol{e}_{z}\right)-\nabla \times(\nabla \times \boldsymbol{B})+\mathrm{Rm} \nabla \times(\boldsymbol{u} \times \boldsymbol{B}) .
\end{aligned}
$$

The flow $\boldsymbol{u}$ is kinematically prescribed as

$\boldsymbol{u}=s \Omega \boldsymbol{e}_{\phi}, \quad$ where $\quad \Omega=\frac{1}{\sqrt{1+\left(s / s_{0}\right)^{2}}}$,

where $s$ is cylindrical radius, and $s_{0}=0.5$. By itself such a flow generates no interaction with the imposed field $\boldsymbol{B}_{0}=\boldsymbol{e}_{z}$, not even a linearly growing toroidal field. It is only with the addition of the Hall term that instabilities may develop in this otherwise stationary basic state.

Investigations of the full field/flow coupling have already been conducted by Wardle (1999) and Balbus \& Terquem (2001) in the local approximation, as well as globally by Rüdiger \& Kitchatinov (2005). These works show that the interaction between the Hall effect and the MRI may lead to both stabilization or destabilization of the system, depending on the values of the

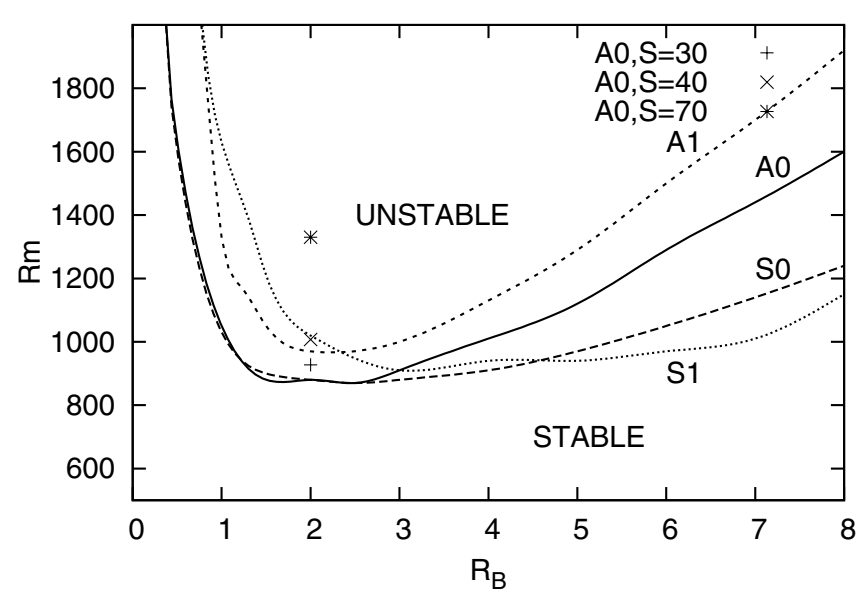

Fig. 1. Stability curves for the axisymmetric (S0 and A0) and nonaxisymmetric ( $\mathrm{S} 1$ and $\mathrm{A} 1$ ) modes. The single points belong to the system where the flow equation was treated in addition to the induction equation.

Lundquist number $\left(S=B_{0} R / \sqrt{(} 4 \pi \rho\right) \eta$, where $\rho$ is the mass density) and $R_{B}$. In the context of Rüdiger \& Kitchatinov (2005), our case corresponds to the limit of small $S$. For example, Fig. 1 illustrates how the increase in $S$ (the influence of the Lorentz force) has a stabilizing effect.

It is also useful to derive the energy equation associated with either Eq. (1) or (2). Taking the dot product of either equation with $\boldsymbol{B}$ and integrating over the volume, one obtains

$$
\frac{\mathrm{d}}{\mathrm{d} t} \int \frac{1}{2} \boldsymbol{B}^{2} \mathrm{~d} V=-\int(\nabla \times \boldsymbol{B})^{2} \mathrm{~d} V+\mathrm{Rm} \int \boldsymbol{B} \cdot \nabla \times(\boldsymbol{u} \times \boldsymbol{B}) \mathrm{d} V .
$$

The Hall term exactly conserves magnetic energy, in both the fully nonlinear and the linearized versions of the induction equation. The only source of energy is from the differential rotation. The Hall term is therefore essentially a catalyst, necessary for the instability to proceed, but energetically incapable of driving instabilities itself (Wareing \& Hollerbach 2009). This situation is exactly analogous to the MRI, where the energy source is also purely from the differential rotation, but a magnetic field is nevertheless necessary. It is, perhaps, not surprising then that the shear-Hall instability, like the MRI, grows on the fast rotational timescale, as we demonstrate below.

According to the local analysis of Urpin \& Rüdiger (2005), for a general differential rotation depending on both $s$ and $z$, a necessary condition for the existence of unstable modes is

$(\boldsymbol{k} \cdot \boldsymbol{B})\left(k_{z} \frac{\partial \Omega}{\partial s}-k_{s} \frac{\partial \Omega}{\partial z}\right)<0$,

where $\boldsymbol{k}$ is the wavenumber. For $\Omega=\Omega(s)$, this reduces to

$B_{z} \frac{\mathrm{d} \Omega}{\mathrm{d} s}<0$,

so that, for a given gradient of the angular velocity, instability only occurs for one sign of $B_{z}$ (Wardle 1999; Balbus \& Terquem 2001). For our particular profile (3) this condition is only fulfilled for positive $B_{z}$, hence $R_{B}$. For negative $R_{B}$ there is no SHI instability.

Because Eq. (2) is linear with an axisymmetric basic state, the different azimuthal modes $\exp (\mathrm{i} m \phi)$ decouple and can be studied separately. Each azimuthal mode further decouples into 
equatorially symmetric and anti-symmetric modes. We will present results for S0, A0, S1, and A1, where "S" or "A" refers to the radial component of the field being equatorially symmetric or anti-symmetric, and the " 0 " or " 1 " refers to the wavenumber $m$.

Equation (2) is solved in a spherical shell using the spherical harmonics code described by Hollerbach (2000). At both $R_{\text {in }}=0.7$ and $R_{\text {out }}=1$ boundaries, the solution smoothly matches a potential external field. At $R_{\text {out }}$ this is indeed the correct condition, but at $R_{\text {in }}$ it is clearly a considerable simplification of the true physics governing the coupling to an internal core field. Resolutions up to 30 Chebyshev polynomials in $r$ and 70 Legendre functions in $\theta$ were used, which was sufficient to resolve the eigenmodes up to $R_{B}=8$. The Hall parameter close to unity is appropriate during the initial stages of a neutron star, and also turns out to be where the shear-Hall instability operates most efficiently.

\section{Results}

\subsection{Stability maps}

Figure 1 shows the instability curves for the four modes S0, $\mathrm{A} 0, \mathrm{~S} 1$, and $\mathrm{A} 1$. Over most of the $R_{B}$ range the axisymmetric modes are slightly preferred over the non-axisymmetric ones, but the curves are otherwise all qualitatively similar. The minimum value of $\mathrm{Rm}$ occurs when $R_{B} \simeq 2-4$. At this minimum the shear frequency $\omega_{\text {sh }}$ is around 400 times the Hall frequency $\omega_{\mathrm{H}}$, consistent with the result of Urpin \& Rüdiger (2005) that the instability only exists for $\left|\omega_{\mathrm{sh}}\right|>\left|\omega_{\mathrm{H}}\right|$.

If $R_{B}$ is smaller than $\simeq 2-4$ the system tends toward the (stable) pure differential rotation case, thus explaining the asymptote $\mathrm{Rm} \rightarrow \infty$ as $R_{B} \rightarrow 0$. At the other end, as $R_{B}$ becomes large the Hall effect increasingly dominates, but as noted above, it is energetically incapable of driving any instability itself, so $\mathrm{Rm}$ must again increase to compensate. For both $R_{B} \ll 1$ and $R_{B} \gg 1$, therefore, the differential rotation must become increasingly strong for the instability to operate.

An intriguing feature of the small $R_{B}$ ends of all four instability curves is that none of them shows any evidence of tilting back over to the right (down to our lowest investigated $R_{B}$ value of 0.4 ), that is, there is no indication of the instability switching off again for sufficiently large $\mathrm{Rm}$. This is perhaps not so surprising for the axisymmetric modes; For example, the classical, axisymmetric MRI has the same feature. For nonaxisymmetric modes, though, it is often the case that a strong enough differential rotation suppresses them again (e.g. Rüdiger et al. 2007; Hollerbach et al. 2010). It is not entirely clear why this does not occur here - or perhaps it does, but only at much higher values of $\mathrm{Rm}$ than what we were able to compute here.

It is also interesting that there is no region where a single mode dominates: both $\mathrm{A}$ and $\mathrm{S}$ modes, and both axisymmetric and non-axisymmetric ones, are excited at comparable parameter values. This suggests that the fully nonlinear regime might consist of a mixture of several modes, which can also be considered as an "oblique rotator". If different modes are indeed present simultaneously, the field configuration and amplitude in the two hemispheres would necessarily be different. If the heat transport is furthermore dominated by the magnetic suppression of the thermal conductivity tensor (Schaaf 1990; Heyl \& Hernquist 2001), then the Hall effect would thereby lead to different temperatures, and hence X-ray emission (Becker \& Pavlov 2002), in the two hemispheres.

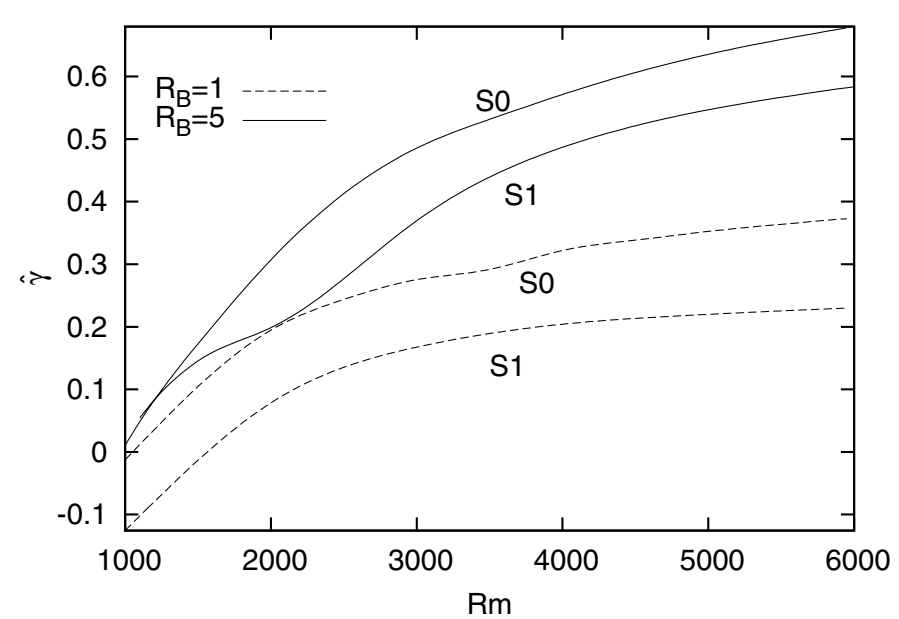

Fig. 2. $\hat{\gamma}$ is a nondimensionalized growth rate $\left(\hat{\gamma}=\tau_{\text {rot }} \gamma\right.$, where $\gamma$ is in physical units), of the S0 and S1 modes, for $R_{B}=1$ and 5. The profiles for $\mathrm{A} 0$ and $\mathrm{A} 1$ are very similar, and are not shown here.

\subsection{Growth rates}

To assess the possible astrophysical significance of the shearHall instability, we need to know not only for what parameter values it exists, but also what its associated growth rates are. Figure 2 shows the growth rate scaled on the rotational timescale. (That is, if $\gamma_{\text {diff }}$ is the growth rate on the chosen diffusive timescale $R^{2} / \eta$, then $\hat{\gamma}=(2 \pi / \mathrm{Rm}) \gamma_{\text {diff }}$ is the growth rate on the rotational timescale $\tau_{\text {rot }}=2 \pi / \Omega_{0}$.) For both $R_{B}=1$ and $R_{B}=5$, for large enough $\mathrm{Rm}$ these growth rates $\hat{\gamma}$ eventually saturate at $\mathrm{O}(1)$ values. As before, for the initial onset, all four modes behave comparably, although the axisymmetric modes do have higher growth rates. There is no preferred equatorial symmetry, because the $\mathrm{S}$ and A modes are very similar. Surprisingly, even though the stability map shows that a nonaxisymmetric mode $\mathrm{S} 1$ is less stable in the $R_{B}$ region from 4 onward, its growth is only stronger near the stability line. For higher Rm, the axisymmetric modes are more dominant.

Of the three timescales that are "obviously" present in the problem, namely the fast rotational timescale $\tau_{\text {rot }}$, the much slower diffusive $\tau_{\text {diff }}=R^{2} / \eta=(\mathrm{Rm} / 2 \pi) \tau_{\text {rot }}$, and Hall $\tau_{\text {Hall }}=$ $\tau_{\text {diff }} / R_{B}$ timescales, the shear-Hall instability thus grows the fastest, and is independent of both $\tau_{\text {diff }}$ and $\tau_{\text {Hall }}$. This is surprising, given that it does clearly depend on both the shear-effect and the Hall effect. On the other hand, as noted above, its energy source is entirely drawn from the differential rotation. In this regard it is quite different from a classical $\alpha \omega$ dynamo, for example, where the growth rate is the geometric mean of $\tau_{\text {rot }}$ and $\tau_{\alpha}$, but both the differential rotation and $\alpha$ also contribute to the energy balance.

\subsection{Wave numbers}

The spatial properties of the eigensolutions are given in Fig. 3. The color map represents the toroidal field structure, while the contours depict the field lines of the poloidal component. Comparing the two plots, at $\mathrm{Rm}=2000$ and 10000 , suggests a transition to smaller scales as $\mathrm{Rm}$ increases. The wavenumber also increases for (turbulent) mean-field dynamos at increasingly supercritical dynamo numbers. However, thanks to the high values of their eddy diffusivity, turbulent dynamos operate with moderate values of $\mathrm{Rm}$. The SHI though may easily operate 


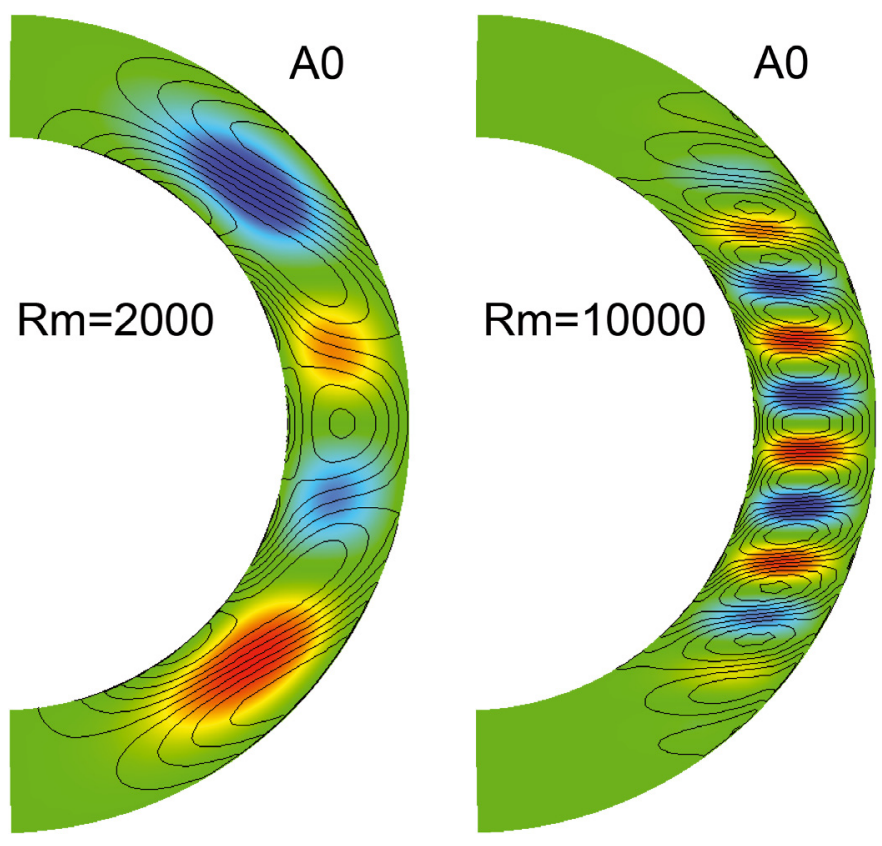

Fig. 3. The axisymmetric solution $\mathrm{A} 0$ for $R_{B}=1$, and $\mathrm{Rm}=2000$ on the left and $\mathrm{Rm}=10000$ on the right. The other modes have the same pattern of exhibiting finer structure in $z$ for larger $\mathrm{Rm}$.

with very high $\mathrm{Rm}$, and may play a dominant role in driving MHD turbulence if $\mathrm{Rm} \gg 1$.

The relative amplitudes of the toroidal field never exceed that of the poloidal field by more than one order of magnitude. Independent of the ratio of the Hall time and the rotation time, the coupling between the components via the Hall term enables their simultaneous, and comparable, amplification.

\section{Discussion}

It remains to be seen to what extent the SHI is important in the regime of newborn neutron stars. We first need to establish the reasonable values for the Hall parameter and the magnetic Reynolds number. It turns out that owing to a low diffusivity $\left(\eta=10^{-(2 . .3)} \mathrm{cm}^{2} \mathrm{~s}^{-1}\right)$ (as treated in the code of Potekhin 2008), the microphysical $\mathrm{Rm}$ is orders of magnitude above the stability line in Fig. 1. If the effects of turbulence are important, according to Naso et al. (2008) diffusivity is much larger and the corresponding $\mathrm{Rm}$ is lower $\left(\sim 10^{3}\right)$, but still supercritical.

The onset of the shear-Hall instability requires a seed field of the magnitude determined by the actual $\mathrm{Rm}$. This field can be provided by various other proposed mechanisms, from fluxconservation to dynamo processes (for an overview, see Spruit 2009). The turbulent $\mathrm{Rm}$ would require $R_{B} \gtrsim 1$, which, for the temperatures of $10^{9 \ldots 10} \mathrm{~K}$ and densities of $10^{10 \ldots 13} \mathrm{~g} \mathrm{~cm}^{-3}$, corresponds to $B \gtrsim 10^{12} \mathrm{G}$. If no turbulence is present, the seed field can be much smaller provided that the stability line remains univalued when $R_{B}$ tends to zero.

In the situation where the magnetic fields are strong, there is a coupling between the fields and the differentially rotating flow, which leads to a stable state of uniform rotation. The timescale for this process is $\tau_{\mathrm{A}}=R \sqrt{4 \pi \rho} / B$, the Alfvén time
(Shapiro 2000), which is only $\sim 10$ s for the neutron star conditions. Is the SHI quick enough to be of any significance? Taking the fast rotation of neutron stars into account, our results (Fig. 2) show that the field can easily amplify several orders of magnitude within the constraining time interval.

Because we are convinced that the instability conditions are fulfilled and the growth is quick enough, there is only the field geometry left to analyze in the context of the standard, inclined magnetic dipole model, which is used to explain the spin-down of pulsars. The SHI generates, as shown, small-scale fields for large $\mathrm{Rm}$. The presence of turbulence is, it seems, a necessary requirement for producing a global field via this instability. On the other hand, X-ray spectra of pulsars sometimes indicate an additional, strong small-scale surface component (Pavlov et al. 2002; Sanwal et al. 2002). It is possible that the SHI is involved in generating the small-scale structure, while the large-scale component should be attributed to another process.

Rapid growth, as well as the easy excitation, indicates that the shear-Hall instability may play an important role in the evolution of the field of newborn neutron stars, prompting further, nonlinear analysis of the field/flow coupling and raising questions about its interaction with other field-generating mechanisms.

\section{References}

Ardeljan, N. V., Bisnovatyi-Kogan, G. S., \& Moiseenko, S. G. 2005, MNRAS, 359,333

Balbus, S. A., \& Terquem, C. 2001, ApJ, 552, 235

Becker, W., \& Pavlov, G. 2002, in The Century of Space Science, Vol. I, ed. J. A Bleeker, J. Geiss, \& M. C. E. Huber (Kluwer), 721

Burrows, A., Dessart, L., Livne, E., Ott, C. D., \& Murphy, J. 2007, ApJ, 664, 416

Choudhuri, A. R., Schüssler, M., \& Dikpati, M. 1995, A\&A, 303, L29

Dall'Osso, S., Shore, S. N., \& Stella, L. 2009, MNRAS, 398, 1869

Dimmelmeier, H., Font, J. A., \& Müller, E. 2002, A\&A, 393, 523

Dudley, M. L., \& James, R. W. 1989, Proc. R. Soc. London, Ser A., 425, 407

Heyl, J. S., \& Hernquist, L. 2001, MNRAS, 324, 292

Hollerbach, R. 2000, Int. J. Numer. Meth. Fluids, 32, 773

Hollerbach, R., \& Rüdiger, G. 2004, MNRAS, 347, 1273

Hollerbach, R., Teeluck, V., \& Rüdiger, G. 2010, Phys. Rev. Lett., 104, 044502

Janka, H.-T., \& Moenchmeyer, R. 1989, A\&A, 226, 69

Kotake, K., Sawai, H., Yamada, S., \& Sato, K. 2004, ApJ, 608, 391

Küker, M., Rüdiger, G., \& Schultz, M. 2001, A\&A, 374, 301

Mönchmeyer, R., \& Müller, E. 1989, in Timing Neutron Stars, ed. H. Ögelman, \& E. P. J. van den Heuvel (Kluwer), 549

Naso, L., Rezzolla, L., Bonanno, A., \& Paternò, L. 2008, A\&A, 479, 167

Ott, C. D., Ou, S., Tohline, J. E., \& Burrows, A. 2005, ApJ, 625, L119

Page, D., Geppert, U., \& Weber, F. 2006, Nucl. Phys. A, 777, 497

Pavlov, G. G., Zavlin, V. E., Sanwal, D., \& Trümper, J. 2002, ApJ, 569, L95

Potekhin, A. Y. 2008, Electron Conductivity of Stellar Plasmas,

http://www . ioffe.ru/astro/conduct/index.html

Rüdiger, G., \& Kitchatinov, L. L. 2005, A\&A, 434, 629

Rüdiger, G., Hollerbach, R., Gellert, M., \& Schultz, M. 2007, AN, 328, 1158

Sanwal, D., Pavlov, G. G., Zavlin, V. E., \& Teter, M. A. 2002, ApJ, 574, L61

Schaaf, M. E. 1990, A\&A, 227, 61

Schmitt, D., Schüssler, M., \& Ferriz-Mas, A. 1996, A\&A, 311, L1

Shapiro, S. L. 2000, ApJ, 544, 397

Spruit, H. C. 2002, A\&A, 381, 923

Spruit, H. C. 2009, in IAU Symp. 259, ed. K. G. Strassmeier, A. G. Kosovichev, \& J. E. Beckman, 61

Urpin, V., \& Rüdiger, G. 2005, A\&A, 437, 23

Vainshtein, S. I., Chitre, S. M., \& Olinto, A. V. 2000, Phys. Rev. E, 61, 4422

Wardle, M. 1999, MNRAS, 307, 849

Wareing, C. J., \& Hollerbach, R. 2009, A\&A, 508, L39 